\title{
Duality for Continuous Graphical Models
}

\author{
Mehdi Molkaraie \\ Department of Statistical Sciences \\ University of Toronto \\ mehdi.molkaraiedalumni.ethz.ch
}

\begin{abstract}
The dual normal factor graph and the factor graph duality theorem have been considered for discrete graphical models. In this paper, we show an application of the factor graph duality theorem to continuous graphical models. Specifically, we propose a method to solve exactly the Gaussian graphical models defined on the ladder graph if certain conditions on the local covariance matrices are satisfied. Unlike the conventional approaches, the efficiency of the method depends on the position of the zeros in the local covariance matrices. The method and details of the dualization are illustrated on two toy examples.
\end{abstract}

\section{INTRODUCTION}

Graphical models represent the decomposition of multivariate functions into the product of several local factors. Usually, each local factor depends on a small subset of the variables.

The normal factor graph duality theorem has been previously applied to computational problems in discrete graphical models (e.g., discrete spin systems and codes on graphs) [1][4]. In this paper, we consider an application of the duality theorem to continuous graphical models. Our focus is on Gaussian graphical models. These models are extremely useful in many different areas, including spatial statistics [5] and gene expression studies [6]. Another reason for the significance of Gaussian graphical models is due to the multivariate central limit theorem [7, Chapter 3]. For more details on Gaussian graphical models, see [8], [9], [10, Chapter 5].

A zero-mean real random vector $\mathbf{X}_{N \times 1}$ has an $N$-variate Gaussian distribution if it has the following PDF

$$
p(\mathbf{x})=\frac{1}{\operatorname{det}(2 \pi \Sigma)^{1 / 2}} \exp \left(-\frac{1}{2} \mathbf{x}^{\top} \Sigma^{-1} \mathbf{x}\right), \quad \mathbf{x} \in \mathbb{R}^{N}
$$

where the symmetric positive-definite matrix $\Sigma^{-1} \in \mathbb{R}^{N \times N}$ is the precision (information) matrix and $\Sigma$ is the corresponding covariance matrix. We will use the notation $\Sigma \succ 0$ to indicate that $\Sigma$ is positive-definite, and sometimes denote the sequence $\left(x_{1}, x_{2}, \ldots, x_{N}\right)$ by $\mathbf{x}_{1}^{N}$.

The structure of a Gaussian graphical model is specified by the precision matrix. Indeed, the nonzero off-diagonal entries of the precision matrix indicate the presence of an edge that connects the two corresponding random variables in the graphical model. Moreover, according to the pairwise Markov property, two non-adjacent random variables are conditionally independent given all the other variables in the model [10].

We consider the problem of solving exactly Gaussian graphical models for a specific model (i.e., the ladder graph) if certain conditions on the local covariance and the local precision matrices are satisfied. By "exactly solve" we mean that the method can efficiently compute the normalization constant in (1), which boils down to an efficient computation of $\operatorname{det}(\Sigma)$. In our framework, we consider the dual normal factor graph (NFG) of the Gaussian ladder graph. Our approach relies on this key property of multivariate Gaussian distributions: in the Fourier transform of a multivariate Gaussian distribution the precision matrix is replaced by the covariance matrix in the exponent [7, Chapter 2].

Contrary to the standard approaches, our method relies on the position of the zeros of the covariance matrices associated with the local factors, where the local covariance matrices are required to have cycle-free graphical representations.

In order to perform exact inference, we first transform the Gaussian graphical model into a cycle-free NFG via the Fourier transformation of the local factors. In cycle-free (Gaussian) graphical models, exact inference can then be done efficiently via the (Gaussian) belief propagation algorithm [11].

\section{THE MODEL}

First, we consider the following PDF defined by the product of $L$ local factors as

$$
p(\mathbf{x})=\frac{1}{Z_{f}} \prod_{\ell=1}^{L} f_{\ell}\left(x_{(\ell-1) k+1}, x_{(\ell-1) k+2}, \ldots, x_{(\ell+1) k}\right),
$$

where $Z_{f}$ is the appropriate normalization constant. Each local factor $f_{\ell}\left(\mathbf{x}_{(\ell-1) k+1}^{(\ell+1) k}\right)$ is given by

$$
\begin{gathered}
f_{\ell}\left(x_{(\ell-1) k+1}, \ldots, x_{(\ell+1) k}\right)= \\
\exp \left(-\frac{1}{2}\left[x_{(\ell-1) k+1}, \ldots, x_{(\ell+1) k}\right] \Sigma_{\ell}^{-1}\left[\begin{array}{c}
x_{(\ell-1) k+1} \\
\vdots \\
x_{(\ell+1) k}
\end{array}\right]\right)
\end{gathered}
$$

for $1 \leq \ell \leq L$.

Here, $\Sigma_{\ell}^{-1}$ is the local precision matrix associated with $f_{\ell}$. In this setup, each local factor contains $2 k$ variables, and for $1 \leq \ell<L$ two consecutive factors $f_{\ell}$ and $f_{\ell+1}$ have exactly $k$ variables $\mathbf{x}_{\ell k+1}^{(\ell+1) k}$ in common. Therefore, the total number of variables in the model $N$ is given by

$$
N=(L+1) k
$$

Clearly $p(\mathbf{x})$ in (2) follows a zero-mean $N$-variate Gaussian distribution. Let

$$
Z_{f}=\operatorname{det}(2 \pi \Sigma)^{1 / 2}
$$

where $\Sigma$ is the covariance matrix associated with $p(\mathbf{x})$. 


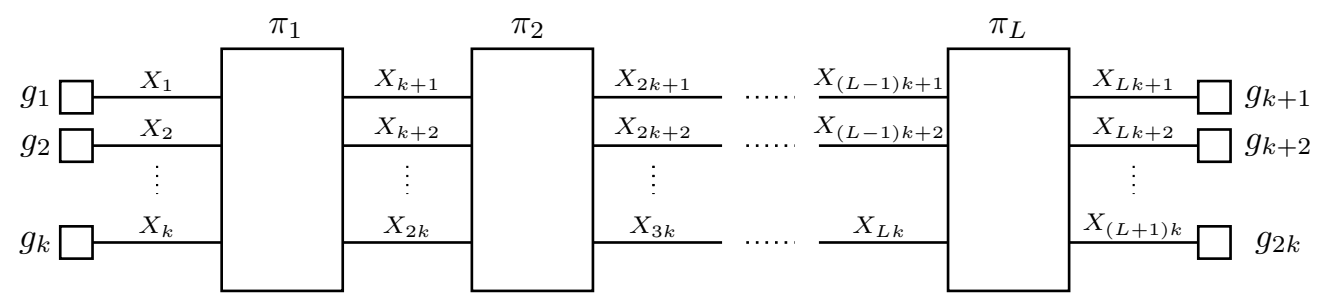

Fig. 1. The NFG for the ladder graph with factorization in [6. The factors $\left\{\pi_{\ell}, 1 \leq \ell \leq L\right\}$ are represented by big boxes and the variables $\left\{X_{i}, 1 \leq i \leq N\right\}$ are represented by edges. The small boxes represent the constant one factors $\left\{g_{i}, 1 \leq i \leq 2 k\right\}$.

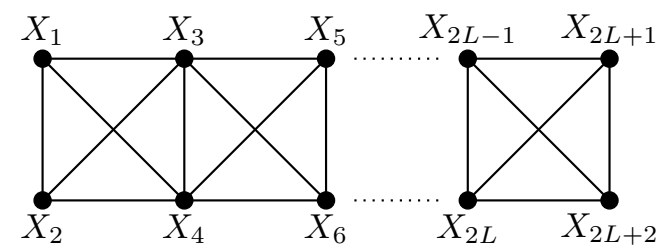

Fig. 2. Dependencies among the variables in Fig. 1 for $k=2$, where all entries of the local precision matrices $\left\{\Sigma_{\ell}^{-1}, 1 \leq \ell \leq L\right\}$ are non-zero.

Next, we write the PDF in (2) in a slightly different form as

$$
\pi(\mathbf{x})=\frac{1}{Z} \prod_{\ell=1}^{L} \pi_{\ell}\left(x_{(\ell-1) k+1}, \ldots, x_{(\ell+1) k}\right),
$$

where $Z$ is the normalization constant, and $\pi_{\ell}\left(\mathbf{x}_{(\ell-1) k+1}^{(\ell+1) k}\right)$

$$
\begin{aligned}
& \pi_{\ell}\left(x_{(\ell-1) k+1}, \ldots, x_{(\ell+1) k}\right)= \\
& \frac{1}{Z_{\ell}} f_{\ell}\left(x_{(\ell-1) k+1}, \ldots, x_{(\ell+1) k}\right)
\end{aligned}
$$

has a zero-mean $2 k$-variate Gaussian distribution for $1 \leq \ell \leq$ $L$. Fom (3), the local normalization constant $Z_{\ell}$ is

$$
Z_{\ell}=\operatorname{det}\left(2 \pi \Sigma_{\ell}\right)^{1 / 2}
$$

The global factorization (6) creates a ladder graph as a concatenation of $L$ blocks (rungs), in which, each block $\pi_{\ell}$ has a zero-mean $2 k$-variate Gaussian distribution.

The normalization constants $Z$ and $Z_{f}$ are closely related. It is straightforward to show that

$$
\begin{aligned}
Z & =\frac{Z_{f}}{\prod_{\ell=1}^{L} Z_{\ell}} \\
& =\frac{\operatorname{det}(2 \pi \Sigma)^{1 / 2}}{\prod_{\ell=1}^{L} \operatorname{det}\left(2 \pi \Sigma_{\ell}\right)^{1 / 2}}
\end{aligned}
$$

Our aim in this paper is to propose an efficient method to compute the exact value of $\operatorname{det}(\Sigma)$, which from (10), boils down to an efficient computation of $Z$. Although the problems of computing $Z_{f}$ and $Z$ essentially have the same computational complexity, it is more convenient to describe our approach in terms of $\pi(\mathbf{x})$ in (6) rather than $p(\mathbf{x})$ in (2).

\section{Assumptions AND GRAPHICAL MODELS}

We suppose the following assumptions hold for the local covariance and the local precision matrices:

I. All local covariance matrices are $2 k \times 2 k$ symmetric positive-definite matrices, i.e.,

$$
\Sigma_{\ell} \succ 0, \quad \forall \ell, 1 \leq \ell \leq L
$$

II. All local precision matrices $\left\{\Sigma_{\ell}^{-1}, 1 \leq \ell \leq L\right\}$ have graphical representations with cycles.

III. All local covariance matrices $\left\{\Sigma_{\ell}, 1 \leq \ell \leq L\right\}$ and their concatenation have cycle-free graphical representations.

We will use NFGs as graphical models [12], [13]. The NFG for the factorization (6) is illustrated in Fig. 1) in which, the big boxes represent the factors $\left\{\pi_{\ell}, 1 \leq \ell \leq L\right\}$ as in $(7)$, the edges represent the variables $\left\{X_{i}, 1 \leq i \leq N\right\}$, and the small boxes represent additional constant one factors $\left\{g_{i}, 1 \leq i \leq 2 k\right\}$. In Fig. 1, we have attached $2 k$ additional univariate constant one factors $\left\{g_{i}, 1 \leq i \leq 2 k\right\}$ to the NFG, $k$ such factors to the leftmost side, and the remaining $k$ factors to the rightmost side of the ladder. Obviously, these extra factors do not affect the value of $Z$, however, including them in the model will facilitate our analysis in Section IV

Alternatively, we can illustrate the dependencies among the variables in Fig. 1 with a more compact graphical representation. By Assumption II, the local precision matrices

$$
\left\{\Sigma_{\ell}^{-1}, 1 \leq \ell \leq L\right\}
$$

have graphical model representations that contain cycles. Let $k=2$, and assume that all entries of the $4 \times 4$ local precision matrices are non-zero. Each block (rung) will therefore have a fully-connected graphical representation (as every pair of distinct variables is connected by an edge). Concatenation of these blocks will then form the ladder graph depicted in Fig. 2 


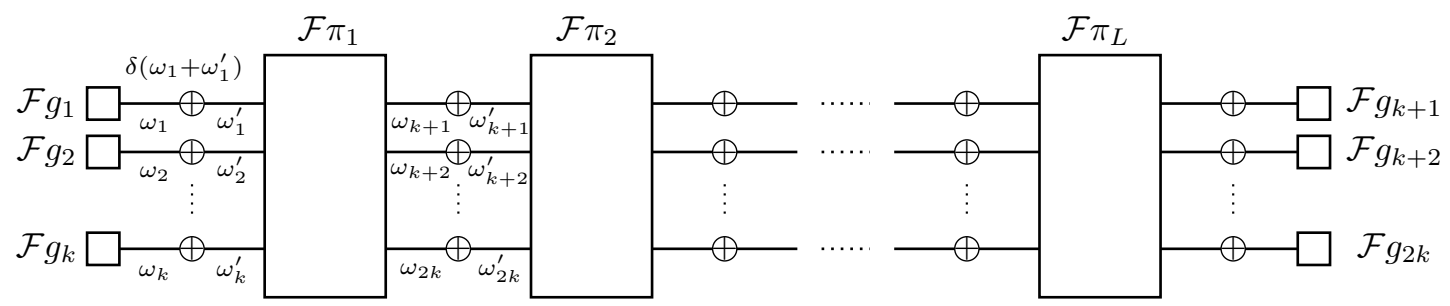

Fig. 3. The dual NFG of Fig. 1 The big boxes represent the factors $\left\{\mathcal{F} \pi_{\ell}, 1 \leq \ell \leq L\right\}$ in 16 , the edges represent the variables $\left\{\boldsymbol{\omega}_{i}, \boldsymbol{\omega}_{i}^{\prime}, 1 \leq i \leq N\right\}$, and the small boxes represent the factors $\left\{\mathcal{F} g_{i}, 1 \leq i \leq 2 k\right\}$ given by [15]. The factors denoted by $\oplus$ transform each edge to a sign-inverting edge.

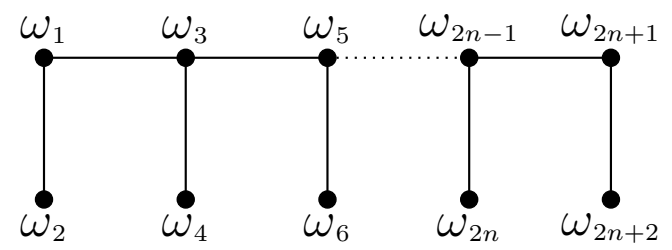

Fig. 4. Dependencies among the variables in Fig. 3 for $k=2$. The local covariance matrices have cycle-free graphical representations with structure as in [19.

It is clear from Figs. 1 and 2 that, in general, the graphical model of (6) may contain many short cycles. Next, we will consider the dual NFG of the Gaussian ladder graph in Fig. 1.

\section{The CyCle-Free DuAL NFG}

Following [14], we can construct the dual of the NFG in Fig. 1 by employing these three steps

- Replace each variable $X_{\ell}$ by the dual variable $\omega_{\ell}$.

- Replace each factor by its Fourier transform.

- Replace each edge by a sign-inverting edge.

The Fourier transform of a function $f(\mathbf{x}): \mathbb{R}^{n} \rightarrow \mathbb{C}$ is the function $(\mathcal{F} f)(\boldsymbol{\omega}): \mathbb{R}^{n} \rightarrow \mathbb{C}$ given by

$$
(\mathcal{F} f)(\boldsymbol{\omega})=\int_{-\infty}^{\infty} f(\mathbf{x}) e^{-\mathrm{i} \boldsymbol{\omega}^{\top} \mathbf{x}} d \mathbf{x}
$$

where $\mathbb{C}$ is the set of complex numbers, $\mathrm{i}=\sqrt{-1}$ denotes the unit imaginary number, and $\boldsymbol{\omega}=\left(\omega_{1}, \omega_{2}, \ldots, \omega_{n}\right)^{\top}$.

The Fourier transform of the constant one factors

$$
\left\{g_{i}, 1 \leq i \leq 2 k\right\}
$$

are Dirac delta functions up to scale. Indeed

$$
\left(\mathcal{F} g_{1}\right)\left(\omega_{1}\right)=2 \pi \delta\left(\omega_{1}\right)
$$

and similarly for $\mathcal{F} g_{2}, \mathcal{F} g_{3}, \ldots, \mathcal{F} g_{2 k}$.

The factors $\mathcal{F} g_{1}, \mathcal{F} g_{2}, \ldots, \mathcal{F} g_{2 k}$ will replace the factors $g_{1}, g_{2}, \ldots, g_{2 k}$ in the dual NFG. Again, $k$ of these factors are connected to the leftmost side, and the remaining $k$ factors to the rightmost side of the dual graph.

The Fourier transform of $\pi_{\ell}$ in (7) is

$$
\begin{gathered}
\left(\mathcal{F} \pi_{\ell}\right)\left(\omega_{(\ell-1) k+1}, \ldots, \omega_{(\ell+1) k}\right)= \\
\exp \left(-\frac{1}{2}\left[\omega_{(\ell-1) k+1}, \ldots, \omega_{(\ell+1) k}\right] \Sigma_{\ell}\left[\begin{array}{c}
\omega_{(\ell-1) k+1} \\
\vdots \\
\omega_{(\ell+1) k}
\end{array}\right]\right)
\end{gathered}
$$

Here $\Sigma_{\ell}$ is the covariance matrix associated with $\pi_{\ell}$. Each factor $\pi_{\ell}$ is then replaced by $\mathcal{F} \pi_{\ell}$ in the dual NFG. The crucial property of the Fourier transform of the multivariate Gaussian distribution is that in $\mathcal{F} \pi_{\ell}$ the covariance matrix is replaced by the precision matrix in the exponent 1

Sign-inverting edges are created by inserting a $\oplus$-factor in the middle of each edge. The $\oplus$-factors will impose the constraint that the addition of their two arguments should be zero in $\mathbb{R}$. The multiplication of all the $\oplus$-factors can be expressed by

$$
\prod_{i=1}^{N} \delta\left(\omega_{i}+\omega_{i}^{\prime}\right),
$$

where $N$ is the total number of variables as in (4).

The dual of the NFG in Fig. 1 is illustrated in Fig. 3, where the big boxes represent $\left\{\mathcal{F} \pi_{\ell}, 1 \leq \ell \leq L\right\}$ as in (16), the edges denote the variables $\left\{\omega_{i}, \omega_{i}^{\prime}, 1 \leq i \leq N\right\}$, the small boxes correspond to the additional factors $\left\{\mathcal{F} g_{i}, 1 \leq i \leq 2 k\right\}$ given by (15), and the $\oplus$-factors in the middle of each edge denote sign-inverters.

The global PDF in the dual domain $\pi^{\prime}$ is given by

$$
\begin{aligned}
& \pi^{\prime}(\boldsymbol{\omega})= \\
& \frac{1}{Z^{\prime}} \prod_{i=1}^{N} \delta\left(\omega_{i}+\omega_{i}^{\prime}\right) \prod_{i=1}^{k}\left(\mathcal{F} g_{i}\right)\left(\omega_{i}\right) \prod_{i=1}^{k}\left(\mathcal{F} g_{k+i}\right)\left(\omega_{L k+i}^{\prime}\right) . \\
& \prod_{\ell=1}^{L}\left(\mathcal{F} \pi_{\ell}\right)\left(\omega_{(\ell-1) k+1}^{\prime}, \ldots, \omega_{\ell k}^{\prime}, \omega_{\ell k+1}, \ldots, \omega_{(\ell+1) k}\right),
\end{aligned}
$$

where $Z^{\prime}$ is the appropriate normalization constant.

In the dual domain, we can again show the dependencies among the variables using a more compact graphical represen-

\footnotetext{
${ }^{1}$ Since $\pi_{\ell}$ follows a zero-mean multivariate Gaussian distribution, its Fourier transform $\mathcal{F} \pi_{\ell}$ is identical to its characteristic function [7].
} 


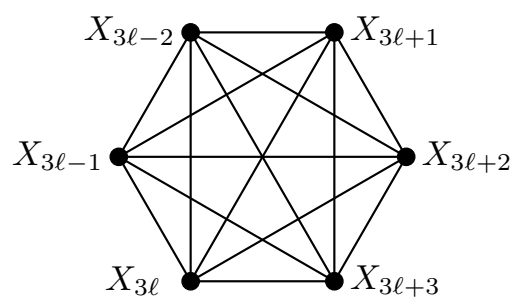

Local Precision Matrix $\Sigma_{\ell}^{-1}$

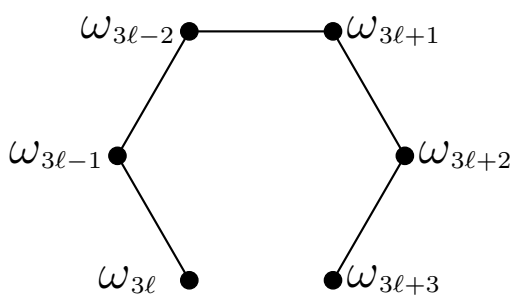

Local Covariance Matrix $\Sigma_{\ell}$

Fig. 5. The contrast between the dependency among the variables associated with the local precision matrix $\Sigma_{\ell}^{-1}$ in the primal domain (left) and among the variables associated with the local covariance matrix $\Sigma_{\ell}$ in the dual domain (right), for $k=3$.

tation. By Assumption III in Section IV the local covariance matrices $\left\{\Sigma_{\ell}, 1 \leq \ell \leq L\right\}$ have cycle-free graphical representations. Set $k=2$, and assume that all the local covariance matrices have the following structure

$$
\Sigma_{\ell}=\left[\begin{array}{llll}
* & * & * & 0 \\
* & * & 0 & 0 \\
* & 0 & * & * \\
0 & 0 & * & *
\end{array}\right]
$$

where $*$ denotes a non-zero real entry. In the corresponding graphical model, each block (rung) and their concatenation are therefore cycle-free - as illustrated in Fig. 4

The factors $\left\{\mathcal{F} g_{i}, 1 \leq i \leq 2 k\right\}$ set the value of $2 k$ out of $N$ independent variables in (18) to zero. Therefore, $\pi^{\prime}$ follows a zero-mean $(N-2 k)$-variate Gaussian distribution. Let us denote the covariance matrix associated with $\pi^{\prime}(\mathbf{x})$ by $\Sigma^{\prime}$. Furthermore, the factors $\left\{\mathcal{F} g_{i}, 1 \leq i \leq 2 k\right\}$ contribute a multiplicative factor of $(2 \pi)^{2 k}$ to $(18)$.

It follows that

$$
\begin{aligned}
Z^{\prime} & =(2 \pi)^{2 k} \operatorname{det}\left(2 \pi \Sigma^{\prime}\right)^{1 / 2} \\
& =(2 \pi)^{k+N / 2} \operatorname{det}\left(\Sigma^{\prime}\right)^{1 / 2}
\end{aligned}
$$

According to the NFG duality theorem, the normalization constants $Z^{\prime}$ and $Z$ are equal up to scale. Indeed

$$
Z^{\prime}=(2 \pi)^{N} Z
$$

For more details, see [13], [14].

Proposition 1. The $\operatorname{det}(\Sigma)$ can be expressed in terms of $\operatorname{det}\left(\Sigma^{\prime}\right)$ and $\left\{\operatorname{det}\left(\Sigma_{\ell}\right), 1 \leq \ell \leq L\right\}$ as

$$
\operatorname{det}(\Sigma)=\operatorname{det}\left(\Sigma^{\prime}\right) \prod_{\ell=1}^{L} \operatorname{det}\left(\Sigma_{\ell}\right)
$$

Proof. The local covariance matrices $\left\{\Sigma_{\ell}, 1 \leq \ell \leq L\right\}$ are of size $2 k \times 2 k$ and $\Sigma$ is of size $N \times N$. Thus, from (4), (10), and (22) we obtain

$$
\begin{aligned}
Z^{\prime} & =(2 \pi)^{N} \frac{\operatorname{det}(2 \pi \Sigma)^{1 / 2}}{\prod_{\ell=1}^{L} \operatorname{det}\left(2 \pi \Sigma_{\ell}\right)^{1 / 2}} \\
& =(2 \pi)^{k+N / 2} \frac{\operatorname{det}(\Sigma)^{1 / 2}}{\prod_{\ell=1}^{L} \operatorname{det}\left(\Sigma_{\ell}\right)^{1 / 2}}
\end{aligned}
$$

Combining (21) and (25) completes the proof.

It is therefore possible to compute $Z^{\prime}$ and $\operatorname{det}\left(\Sigma^{\prime}\right)$ exactly (e.g., via the Gaussian belief propagation algorithm [11]) in the cycle-free NFG as in Fig. 3. After this step, we can compute $\operatorname{det}(\Sigma)$ from (23).

We conclude that computing the exact value of $\operatorname{det}(\Sigma)$ in ladder graphs is straightforward in the dual factor graph if all local covariance matrices are "known" to have cycle-free NFGs. On the contrary, if the local precision matrices have cycle-free graphical representations, computing $\operatorname{det}(\Sigma)$ is easy in the primal factor graph. A special case of interest is when the local precision and covariance matrices are diagonal. In this case, computing $\operatorname{det}(\Sigma)$ is easy in both domains.

For $k=3$, the contrast between the dependency among the variables in Figs. 2] and 4 is depicted in Fig. 5. By Assumption II, Fig. 5 (left) has cycles, while, by Assumption III, Fig. 5 (right) is singly-connected.

\section{NumERICAL EXAMPLES}

In the first toy example, we set $k=2$ and $L=3$. For $1 \leq \ell \leq 3$, let

$$
\Sigma_{\ell}=\left[\begin{array}{llll}
2 & 1 & 1 & 0 \\
1 & 2 & 0 & 0 \\
1 & 0 & 2 & 1 \\
0 & 0 & 1 & 2
\end{array}\right]
$$

where

$$
\Sigma_{\ell}^{-1}=\left[\begin{array}{cccc}
1.2 & -0.6 & -0.8 & 0.4 \\
-0.6 & 0.8 & 0.4 & -0.2 \\
-0.8 & 0.4 & 1.2 & -0.6 \\
0.4 & -0.2 & -0.6 & 0.8
\end{array}\right]
$$

with $\operatorname{det}\left(\Sigma_{\ell}\right)=5$. Here $\Sigma_{\ell}$ and $\Sigma_{\ell}^{-1}$ satisfy all the required assumptions discussed in Section [II] This toy example consists of three blocks, where each block has a four-variate Gaussian distribution with a fully-connected NFG. 

by

From (18) the global PDF in the dual domain $\pi^{\prime}(\boldsymbol{\omega})$ is given

$$
\begin{gathered}
\pi^{\prime}(\boldsymbol{\omega})= \\
\frac{1}{Z^{\prime}} \mathcal{F} g_{1}\left(\omega_{1}\right) \mathcal{F} g_{2}\left(\omega_{2}\right) \mathcal{F} g_{3}\left(\omega_{7}^{\prime}\right) \mathcal{F} g_{4}\left(\omega_{8}^{\prime}\right) \prod_{i=1}^{8} \delta\left(\omega_{i}+\omega_{i}^{\prime}\right) . \\
\mathcal{F} \pi_{1}\left(\omega_{1}^{\prime}, \omega_{2}^{\prime}, \omega_{3}, \omega_{4}\right) \mathcal{F} \pi_{2}\left(\omega_{3}^{\prime}, \omega_{4}^{\prime}, \omega_{5}, \omega_{6}\right) \mathcal{F} \pi_{3}\left(\omega_{5}^{\prime}, \omega_{6}^{\prime}, \omega_{7}, \omega_{8}\right)
\end{gathered}
$$

Thus

$$
\begin{aligned}
& Z^{\prime}=(2 \pi)^{4} \\
& \int_{\boldsymbol{\omega}} \delta\left(\omega_{1}\right) \delta\left(\omega_{2}\right) \delta\left(\omega_{7}^{\prime}\right) \delta\left(\omega_{8}^{\prime}\right) \prod_{i=1}^{8} \delta\left(\omega_{i}+\omega_{i}^{\prime}\right) \cdot \mathcal{F} \pi_{1} \mathcal{F} \pi_{2} \mathcal{F} \pi_{3} d \boldsymbol{\omega}
\end{aligned}
$$

which gives

$$
\begin{gathered}
Z^{\prime}=(2 \pi)^{4} \int_{\omega_{3}, \omega_{4}, \omega_{5}, \omega_{6}} \mathcal{F} \pi_{1}\left(0,0, \omega_{3}, \omega_{4}\right) \\
\mathcal{F} \pi_{2}\left(-\omega_{3},-\omega_{4}, \omega_{5}, \omega_{6}\right) \mathcal{F} \pi_{3}\left(-\omega_{5},-\omega_{6}, 0,0\right) d \omega_{3} d \omega_{4} d \omega_{5} d \omega_{6}
\end{gathered}
$$

which after substituting $\Sigma$ from (26) yields

$$
\begin{aligned}
& Z^{\prime}=(2 \pi)^{4} \\
& \int_{\omega_{3}, \omega_{4}, \omega_{5}, \omega_{6}} \exp \left(-\frac{1}{2}\left[\omega_{3}, \omega_{4}, \omega_{5}, \omega_{6}\right] \Sigma^{\prime-1}\left[\begin{array}{l}
\omega_{3} \\
\omega_{4} \\
\omega_{5} \\
\omega_{6}
\end{array}\right]\right) d \omega_{3} d \omega_{4} d \omega_{5} d \omega_{6}
\end{aligned}
$$

with

$$
\Sigma^{\prime-1}=\left[\begin{array}{cccc}
4 & 2 & -1 & 0 \\
2 & 4 & 0 & 0 \\
-1 & 0 & 4 & 2 \\
0 & 0 & 2 & 4
\end{array}\right]
$$

To compute $\operatorname{det}\left(\Sigma^{\prime}\right)$, we can either apply the Gaussian belief propagation algorithm, or, in this toy example, directly compute

$$
\operatorname{det}\left(\Sigma^{\prime}\right)=1 / 128
$$

From 23) we have

$$
\operatorname{det}(\Sigma)=125 / 128
$$

where $\Sigma$ is the $8 \times 8$ covariance matrix associated with the eight-variate Gaussian distribution in the primal NFG.

In our second example, we set $k=3$ and $L=2$. Let

$$
\Sigma_{1}=\left[\begin{array}{llllll}
3 & 1 & 0 & 2 & 0 & 0 \\
1 & 2 & 1 & 0 & 0 & 0 \\
0 & 1 & 3 & 0 & 0 & 0 \\
2 & 0 & 0 & 3 & 1 & 0 \\
0 & 0 & 0 & 1 & 2 & 1 \\
0 & 0 & 0 & 0 & 1 & 3
\end{array}\right]
$$

in the first block, where

$$
\Sigma_{1}^{-1}=\frac{1}{22}\left[\begin{array}{cccccc}
30 & -18 & 6 & -25 & 15 & -5 \\
-18 & 24 & -8 & 15 & -9 & 3 \\
6 & -8 & 10 & -5 & 3 & -1 \\
-25 & 15 & -5 & 30 & -18 & 6 \\
15 & -9 & 3 & -18 & 24 & -8 \\
-5 & 3 & -1 & 6 & -8 & 10
\end{array}\right]
$$

and $\operatorname{det}\left(\Sigma_{1}\right)=44$. In the second block, let

$$
\Sigma_{2}=\left[\begin{array}{cccccc}
4 & 1 & 0 & -2 & 0 & 0 \\
1 & 3 & -1 & 0 & 0 & 0 \\
0 & -1 & 1 & 0 & 0 & 0 \\
-2 & 0 & 0 & 4 & 1 & 0 \\
0 & 0 & 0 & 1 & 3 & -1 \\
0 & 0 & 0 & 0 & -1 & 1
\end{array}\right]
$$

where

$$
\Sigma_{2}^{-1}=\frac{1}{33}\left[\begin{array}{cccccc}
14 & -7 & -7 & 8 & -4 & -4 \\
-7 & 20 & 20 & -4 & 2 & 2 \\
-7 & 20 & 53 & -4 & 2 & 2 \\
8 & -4 & -4 & 14 & -7 & -7 \\
-4 & 2 & 2 & -7 & 20 & 20 \\
-4 & 2 & 2 & -7 & 20 & 53
\end{array}\right]
$$

and $\operatorname{det}\left(\Sigma_{2}\right)=33$. The covariance matrix $\Sigma$ is $9 \times 9$, and the model consists of two blocks, where each block has a six-variate Gaussian distribution with a fully-connected NFG.

The global PDF in the dual domain is given by

$$
\begin{aligned}
& \pi^{\prime}(\boldsymbol{\omega})=\frac{1}{Z^{\prime}} \prod_{i=1}^{9} \delta\left(\omega_{i}+\omega_{i}^{\prime}\right) . \\
& \mathcal{F} g_{1}\left(\omega_{1}\right) \mathcal{F} g_{2}\left(\omega_{2}\right) \mathcal{F} g_{3}\left(\omega_{3}\right) \mathcal{F} g_{4}\left(\omega_{7}^{\prime}\right) \mathcal{F} g_{5}\left(\omega_{8}^{\prime}\right) \mathcal{F} g_{6}\left(\omega_{9}^{\prime}\right) . \\
& \mathcal{F} \pi_{1}\left(\omega_{1}^{\prime}, \omega_{2}^{\prime}, \omega_{3}^{\prime}, \omega_{4}, \omega_{5}, \omega_{6}\right) \mathcal{F} \pi_{2}\left(\omega_{4}^{\prime}, \omega_{5}^{\prime}, \omega_{6}^{\prime}, \omega_{7}, \omega_{8}, \omega_{9}\right)
\end{aligned}
$$

After a little manipulation

$$
\begin{aligned}
& Z^{\prime}=(2 \pi)^{6} \\
& \int_{\omega_{4}, \omega_{5}, \omega_{6}} \exp \left(-\frac{1}{2}\left(7 \omega_{4}^{2}+5 \omega_{5}^{2}+4 \omega_{6}^{2}+4 \omega_{4} \omega_{5}\right)\right) d \omega_{4} d \omega_{5} d \omega_{6}
\end{aligned}
$$

It is then easy to compute $\operatorname{det}\left(\Sigma^{\prime}\right)=1 / 132$. Therefore, from (23) we obtain

$$
\operatorname{det}(\Sigma)=\frac{44 \times 33}{132}=11
$$

In general, the cycle-free Gaussian NFG of $\pi^{\prime}(\boldsymbol{\omega})$ in 18 consists of unary factors (attached to the vertices) and pairwise factors (placed on the edges). For larger values of $N$, we can employ the Gaussian belief propagation algorithm [11] to compute the exact value of $\operatorname{det}\left(\Sigma^{\prime}\right)$.

\section{FUTURE WORK}

Some directions for future work include: i) applying the method to the multivariate Cauchy distribution (or other multivariate distributions) defined on the ladder graph, with assumptions made on the local dispersion matrices, ii) extending the results and using the Gaussian belief propagation in the dual of Gaussian Markov random fields. According to our assumptions in Section III, each building block in the dual NFG of the model is cycle-free, however, arranging these cycle-free blocks horizontally and vertically will create an NFG with cycles, and iii) Comparing the method with existing algorithms on computing the determinant of block-tridiagonal matrices (see, e.g., [15], [16]). Note that the precision matrices associated with ladder graphs are block-tridiagonal by construction. 


\section{CONCLUSION}

We showed an application of the NFG duality theorem to continuous graphical models. A method was proposed to solve exactly the Gaussian graphical models defined on the ladder graph if the local covariance matrices and their concatenation have cycle-free graphical representations.

\section{REFERENCES}

[1] M. Molkaraie and H.-A. Loeliger, "Partition function of the Ising model via factor graph duality," Proc. 2013 IEEE Int. Symp. on Inf. Theory Istanbul, Turkey, July 7-12, 2013, pp. 2304-2308.

[2] A. Al-Bashabsheh and Y. Mao, "On stochastic estimation of the partition function," Proc. 2014 IEEE Int. Symp. on Inf. Theory, Honolulu, USA, June 29 - July 4, 2014, pp. 1504-1508.

[3] G. D. Forney, Jr., "Codes on graphs: duality and MacWilliams identities," IEEE Trans. Inf. Theory, vol. 57, pp. 1382-1397, Feb. 2011.

[4] M. Molkaraie and V. Gómez, "Monte Carlo methods for the ferromagnetic Potts model using factor graph duality," IEEE Trans. Inf. Theory vol. 64, pp. 7449-7464, Dec. 2018.

[5] J. Besag, "Spatial interaction and the statistical analysis of lattice systems," Journal of the Royal Stat. Soc.: Series B, vol. 36, pp. 192-225, Jan. 1974.

[6] A. Dobra, C. Hans, B. Jones, J. R. Nevins, G. Yao, and M. West, "Sparse graphical models for exploring gene expression data," Journal of Multivariate Analysis, vol. 90, pp. 196-212, July 2004.

[7] T. W. Anderson, An Introduction to Multivariate Statistical Analysis. Wiley, 1958.
[8] A. P. Dempster, "Covariance selection," Biometrics, pp. 157-175, March 1972.

[9] H. Rue and L. Held, Gaussian Markov Random Fields: Theory and Applications. CRC Press, 2005.

[10] S. L. Lauritzen, Graphical Models. Oxford University Press, 1996.

[11] Y. Weiss and W. T. Freeman, "Correctness of belief propagation in Gaussian graphical models of arbitrary topology," Neural Computation, vol. 13, pp. 2173-2200, Oct. 2001.

[12] G. D. Forney, Jr., "Codes on graphs: normal realizations," IEEE Trans. Inf. Theory, vol. 47, pp. 520-548, Feb. 2001.

[13] A. Al-Bashabsheh and Y. Mao, "Normal factor graphs and holographic transformations," IEEE Trans. Inf. Theory, vol. 57, pp. 752-763, Feb. 2011.

[14] G. D. Forney, Jr., "Graphical models for elementary algebraic topology, with applications to statistical physics and codes on graphs," IEEE Trans. Inf. Theory, vol. 64, pp. 7465-7487, Dec. 2018.

[15] G. Meurant, "A review on the inverse of symmetric tridiagonal and block tridiagonal matrices," SIAM Journal on Matrix Analysis and Applications, vol. 13, pp. 707-728, July 1992.

[16] L. G. Molinari, "Determinants of block tridiagonal matrices," Linear Algebra and Its Applications, vol. 429, pp. 2221-2226, July 2008. 